\title{
When Posting Is Believing
}

\section{Adaptation and Internalization of Expressed Opinions in Social Network Sites}

\author{
Stephan Winter ${ }^{1} \oplus$, Paola Remmelswaal², and Anne Vos $^{2}$ \\ ${ }^{1}$ Department of Psychology, University of Koblenz-Landau, Germany \\ ${ }^{2}$ Amsterdam School of Communication Research, University of Amsterdam, The Netherlands
}

\begin{abstract}
Social network sites (SNS) facilitate the expression of users' opinions to a large audience. This research aimed to investigate whether the characteristics of this new media context strengthen the adaptation of opinions to the majority and lead to an internalization of the expressed views. Based on literature on public self-presentation and identity shifts, it was assumed that the publicness of and the identifiability within SNS elicit stronger expression effects than online forums or non-public settings. A between-subjects experiment $(N=302)$ varied the visible majority opinion on a news issue as well as the media context in which participants were asked to write down their opinion. Results showed significant adaptation effects to the majority (positive vs. negative comments) across media contexts. The internalization of attitudes was stronger in SNS groups with a more relevant audience but also occurred in other settings. Consequences for the formation of public opinion are discussed.
\end{abstract}

Keywords: social network sites, opinion expression, self-effects, expression effects, experiment

New communication technologies have made it easy to express one's opinion to a large number of people. Posting, commenting, or sharing have become popular features among Internet users, and social network sites (SNS) such as Facebook are increasingly used as platforms of discussion and debate (Thorson \& Wells, 2016). Participatory activities and the creation of content can thus be regarded as central aspects of SNS usage, but the way in which people express their opinion in this new media context and the consequences of such behavior are not yet well understood.

First, the technical ease of opinion expression does not imply that everyone reveals their private attitude and participates in an open debate. Due to considerations of others' views and behavior or expected negative reactions when voicing unpopular views, people are likely to adapt their expressed opinion to the perceived majority (Cialdini \& Goldstein, 2004; Noelle-Neumann, 1974). Second, the act of expressing an opinion publicly may have effects on the sender her-/himself. Research on public self-presentation and identity shifts (e.g., Gonzales \& Hancock, 2008; Tice, 1992) suggests that people internalize publicly displayed behavior even if it did not mirror their own personality or views in the first place. While previous research focused on effects that messages have on their recipients, such effects on the sender of messages that might even be greater remained largely unexplored (Valkenburg, 2017).
Against this background, the present research is intended to explore the question of how people adapt and internalize their expressed opinions in different media contexts. The primary focus lies on SNS but we compare this context with other genres of computer-mediated communication (CMC), namely, online forums (as an earlier and more anonymous genre) and non-public text documents, to analyze whether potential effects are SNS-specific or more general. Given that acts of opinion expression in SNS are tied to one's personal profile and are directed to a large and personally relevant audience (Hogan, 2010), it can be expected that the aforementioned adaptation and expression effects are stronger in SNS settings (especially when expecting future interaction with the members of this network) than in online forums (or non-public forms of opinion expression). One consequence that would be undesirable from a normative understanding of democracy (Steenbergen et al., 2003) could be that SNS usage accelerates the solidification of majority opinions when people predominantly express popular views and the act of expression makes them even stronger. If some users are only exposed to homogeneous communication networks (echo chambers; Boutyline \& Willer, 2017), the visible majority opinion might be an extreme opinion by a specific group - internalization of expressed opinions could then lead to an increase of polarization. 


\section{Adaptation of Opinions: Social Influence of the Majority}

When making sense of a current societal debate or an issue in the news, people tend to base their judgments not only on the merits of the available arguments but also on the opinions of others. A large body of research in social psychology (see Cialdini \& Goldstein, 2004) established the phenomenon of social influence in ambiguous situations. According to Deutsch and Gerard (1955), this influence can be informative (when people use others' behavior or statements as a meaningful cue to make sense of their environment) and/or normative (when people follow a group norm even if they privately think something different).

Concerning the scenario of reading online comments by others, research has shown that observers' own (private) attitudes are influenced by the valence of these comments (e.g., Lee \& Jang, 2010), which can be interpreted as informative social influence. These effects become more likely when the quality of comments (e.g., argument strength) is higher and when people have not yet formed a strong opinion on the topic (Winter et al., 2015). With regard to normative influence, when it comes to opinion expression, private attitudes that are already in line with the perceived majority opinion can be voiced without expectations of negative reactions. However, when people perceive themselves to be in the minority, they may adapt their expressed opinion to the majority (McFarland et al., 1984) - or they may decide to remain silent, as spiral of silence theory predicts for discussions on value-laden topics (Noelle-Neumann, 1974).

The normative part of social influence in particular is likely to depend on characteristics of the media context in which the act of opinion expression takes place. While non-public or anonymous channels allow for a less restricted expression that largely ignores social consequences, others' opinions and their assumed behavior become more important when opinion expression is connected to one's name. As a subcategory of social media (Bayer et al., 2020), SNS, defined as web-based platforms that include (semi-)public profiles, display connections between users and allow for the creation and sharing of user-generated content (Boyd \& Ellison, 2007), constitute a setting in which people (typically) reveal their real name. One key difference to online forums (as an earlier form of CMC) is the level of identifiability (Spears \& Lea, 1994): SNS posts that include opinion expression can be traced back to the originator and can thus be linked to one's identity. Forums also include user-generated content but there is less emphasis on the profile and users are typically less identifiable due to nicknames and a lack of photos, which may provide a greater sense of self-anonymity ("a sender's perceived anonymity to others when he or she is the message source"; Anonymous, 1998, p. 388). In terms of the heuristic-systematic model of persuasion (Chaiken et al., 1996), the conditions of accountability toward relevant interaction partners in SNS are assumed to trigger impression motivation, which was described as a "desire to express attitudes that will be socially acceptable to potential evaluators, both real and imagined" (Chaiken et al., 1989, p. 235). Along these lines, people have been shown to be particularly afraid of negative reactions or evaluations when stating unpopular views on Facebook (Neubaum \& Krämer, 2018). The goal of positive self-presentation that is salient in SNS settings (Nadkarni \& Hofmann, 2012) is thus expected to induce a stronger orientation toward the majority than in media contexts in which users are less identifiable. The following hypotheses are posited:

Hypothesis 1 (H1): The majority opinion in statements of peers affects the valence of people's expressed opinion.

Hypothesis 2 (H2): The adaptation of the expressed opinion toward the majority opinion is stronger in SNS than in online forums or nonpublic settings.

\section{Internalization of Opinions: Expression Effects}

Beyond the influence of peer statements on the formation of attitudes or the decision on what to say or write in a public situation, the act of expression may have an effect on the sender her- or himself. While communication models typically deal with effects on message recipients, the increased opportunities for citizens to become producers of content in SNS also lends importance to effects on message senders. Pingree (2007) was among the first scholars to add this perspective to models of communication research and distinguished the stages of expecting expressions, composing a message, and releasing the message publicly. While the (imagined) audience can already be impactful in the first two stages, an actual expression effect is possible when the sender has publicly expressed her-/himself or posted something online. Valkenburg (2017) recently coined the term "self-effect" and defined it as the phenomenon that "message creators/senders involuntarily influence their own cognitions, emotions, attitudes, or behavior" (p. 478).

Although expression or self-effects have been neglected in prior models of communication research, there are some earlier empirical studies that document changes in message senders' self-concepts or attitudes. For instance, classic studies on forced compliance (Festinger \& Carlsmith, 1959) or counter-attitudinal advocacy (Schlenker \& Goldman, 1982) show that people adjust attitudes to avoid inconsistencies with previous behavior. According to the concept of shared reality (Higgins, 1992), interpersonal 
communication is an attempt to create a common understanding of the environment with meaningful others: Studies in this theoretical realm showed so-called saying-isbelieving effects in the sense that people re-narrate ambiguous stories in a way that is in line with the expectations of the audience and also remember them in this way (e.g., Echterhoff et al., 2008). A related line of research showed that perceptions of oneself can also be affected by induced behavior in public (Baumeister \& Tice, 1984). In a study by Tice (1992), people who had the task to present extraverted versus introverted facets of their personality evaluated themselves more in line with their task - as long as they assumed that they would be observed by others.

In the realm of CMC, Gonzales and Hancock (2008) showed similar effects for public self-presentation online: Those who had the task to portray themselves as outgoing in a public blog post reported a more extraverted selfperception in a personality questionnaire afterwards than those who had portrayed themselves as shy. This effect, termed "identity shift," occurred in the public blog setting but not when participants wrote down their description in a private text-processing document. Walther et al. (2011) replicated and extended these findings by showing that participants' identity shift in public self-presentation is reinforced by positive feedback of others. According to a study by Carr and Foreman (2016), identity shift is particularly amplified by positive feedback by close SNS friends.

The theoretical explanations for identity shifts include assumptions on self-perception (people infer their traits as observers of their own behavior; Bem, 1972) and public commitment (people feel bound to behavior they showed to others; Schlenker et al., 1994). While these explanations are not mutually exclusive, empirical findings on the importance of publicness both offline and online (e.g., Gonzales \& Hancock, 2008; Tice, 1992) predominantly support the notion of public commitment. This suggests that a relevant audience strengthens shifts in message creators' views; private self-reflection may also induce shifts (e.g., effects of diary writing; see Valkenburg, 2017) but can be neglected more easily. Tice (1992) specified further conditions that amplify the internalization of public behavior: (a) the person has a high degree of choice in the content of their selfpresentation, (b) the act of self-presentation is tied to one's identity, and (c) there is a high likelihood of future (face-toface) interaction with the audience. Empirically, the effects of Components $b$ and $c$ were corroborated by findings of increased internalization when participants presented selfportrayals that were their own responses (vs. given statements) and when they believed that they would meet the observer again in the future (Tice, 1992). It can be argued that Condition a has not been fully given in experimental settings in which people were asked to show a certain behavior, therefore, an experimental setting in which participants are asked to express an opinion without giving the direction may be more realistic.

Most prior studies employed personality traits, but the assumptions of self-effects also apply for public opinion expression. In an initiating study, Johnson and van der Heide (2015) investigated the private versus public sharing of media content (with the example of abstract photographs) and only found limited effects of publicness but significant effects of feedback on sharers' liking of the content. A focus on opinions on current affairs seems suitable to extend the scope of empirical research on online identity shifts and to contribute to our understanding of citizens' participation in new media environments. Thereby, our focus lies on opinion shifts or an internalization of expressed opinions. Such an internalization would mean that the sender's private attitude moves further into the direction of the view that is expressed in the statement (e.g., when a person who is moderately in favor of a specific proposition becomes more positive about it after releasing a positive message). As SNS profiles are connected to one's identity (Hogan, 2010) and one's posts are accessible for a relevant audience, we expect that:

Hypothesis 3 (H3): Expressing an opinion in SNS leads to stronger internalization of this opinion compared to expressing an opinion in an online forum or a nonpublic context.

Additionally, concerning the expectation of future interaction (Tice, 1992), it can be assumed that the composition of interaction partners within SNS contexts further affects the extent of internalization:

Hypothesis 4 (H4): The internalization of the expressed opinion is stronger in SNS groups with a higher (vs. lower) likelihood of future interaction.

\section{Method}

An experiment was designed to test the proposed hypotheses. Participants were randomly assigned to one of the 12 conditions of a $4 \times 3$ between-subjects design in which the media context (SNS with low likelihood of future interaction vs. SNS with high likelihood of future interaction vs. online discussion forum vs. text document) and the valence of the presented majority opinion (positive vs. negative vs. no majority opinion) were systematically varied. Despite the advantages in external validity that a field observation would include, we opted for a laboratory experiment as a first program-building study in order to demonstrate potential effects under carefully controlled conditions, to allow for thorough debriefings, and to avoid interference in actual SNS user behavior that may affect nonparticipants. 
The study was approved by the local ethics committee. Data files and materials are available at the OSF repository: https://osf.io/5qf2r

\section{Sample}

Participants were recruited on the campus of the University of Amsterdam (UvA). The requirements to participate in this study were the status of a Communication Science Bachelor student at the UvA (as one manipulation for the SNS conditions was focused on this group, see next section) and the possession of a personal Facebook profile. Participants received 10 Euro or course credits. In total, 307 participants completed the experiment. Five participants were excluded from the final sample because they experienced technical difficulties or could not access their Facebook account on the lab computer, leaving a final sample of 302 participants (220 female; age: $M=21.02, S D=2.48$ ). Prior studies on identity shifts revealed medium-to-large effect sizes (e.g., Carr \& Foreman, 2016: $r=.28$; estimated values of Gonzales \& Hancock, 2008: $f=.28$; estimated values of Tice, 1992: $f=.55$ ). When calculating a post hoc power analysis with the effect size of $f=.28$ and the $4 \times$ 3 design in an ANOVA logic, $G^{*}$ Power showed a sample size of 273 for a power of $95 \%$, which would be covered by the existing sample of 302 .

\section{Stimulus Material}

In the experiment, participants were asked to express their opinion in a discussion on the topic of a planned ban on bicycle-sharing systems in Amsterdam. According to a pilot study (see online Appendix in OSF), the topic was relatively interesting and relevant for the target group and participants' prior attitudes were moderate. For the main experiment, two news articles were written on the bicycle-sharing issue. The articles contained arguments both in favor of and against the bicycle-sharing ban and were printed in the design of an international news website (595 words; Flesch Reading Ease [FRE]; Flesch, 1948; score: 49.6 college level) and a regional newspaper (659 words; FRE score: 44.3). We opted for two articles to provide participants with an overview of the topic and to avoid the potential bias of only one specific source or article.

\section{Procedure}

In the beginning, participants received the two news articles on the topic. Then, participants were asked to read the instructions and to go to their assigned medium to read a poll, a post, and comments about the issue. Participants subsequently answered questions about their attitude toward the topic (to measure the influence of others' comments before opinion expression) and were then redirected to the medium once again to write down their opinion. Hereafter, participants were asked to continue the questionnaire in which they first, as a distraction, had to evaluate the news articles that they read, followed by filler questions measuring their attitude toward other topics such as bonuses for health data. Next, participants' attitude toward the bicycle-sharing issue was measured for a second time, followed by manipulation checks. Then, personality characteristics such as self-monitoring tendencies, participants' SNS usage and motivations, as well as interest in the topic (and prior usage of bikes and sharing systems) were assessed. In the end, participants were fully debriefed.

\section{Independent Variable: Media Context}

Depending on their condition, participants took part in the discussion on either an SNS, an online discussion forum, or a text document. Since the target group consisted of Communication Science Bachelor students of the UvA, a Facebook group with students of this subject reflected the SNS condition with high likelihood of future interaction (see Figure 1), while a Facebook group with students of all Bachelor programs of the UvA reflected the SNS condition with a lower likelihood of future interaction. The online discussion forum was specified as "The online student board," an online forum on which its members can post without their real name and visually anonymously. Finally, a Word document was used to represent the private text document condition (see Gonzales \& Hancock, 2008).

In the SNS conditions, participants were first asked to log in with their personal Facebook profile. In the next step, depending on their condition, they were asked to join one of the two Facebook groups and to carefully study the group page with its poll, posts, and comments. Both Facebook groups were created for the purpose of this study and filled with more than 250 profiles to recreate a realistic student Facebook group. In the forum condition, participants were directed to the online discussion forum and asked to read the poll, posts, and comments on the bicycle-sharing issue. In the text document condition, others' opinions were presented as quotes from students in a Microsoft Word file. Participants in the forum condition were able to comment by using a nickname, while participants in the text document condition were able to comment without using any name or nickname at all.

\section{Independent Variable: Valence of the Majority Opinion}

Participants were exposed to a positive, negative, or no majority opinion toward the topic. In the conditions in which a majority opinion was absent, a neutral post was 


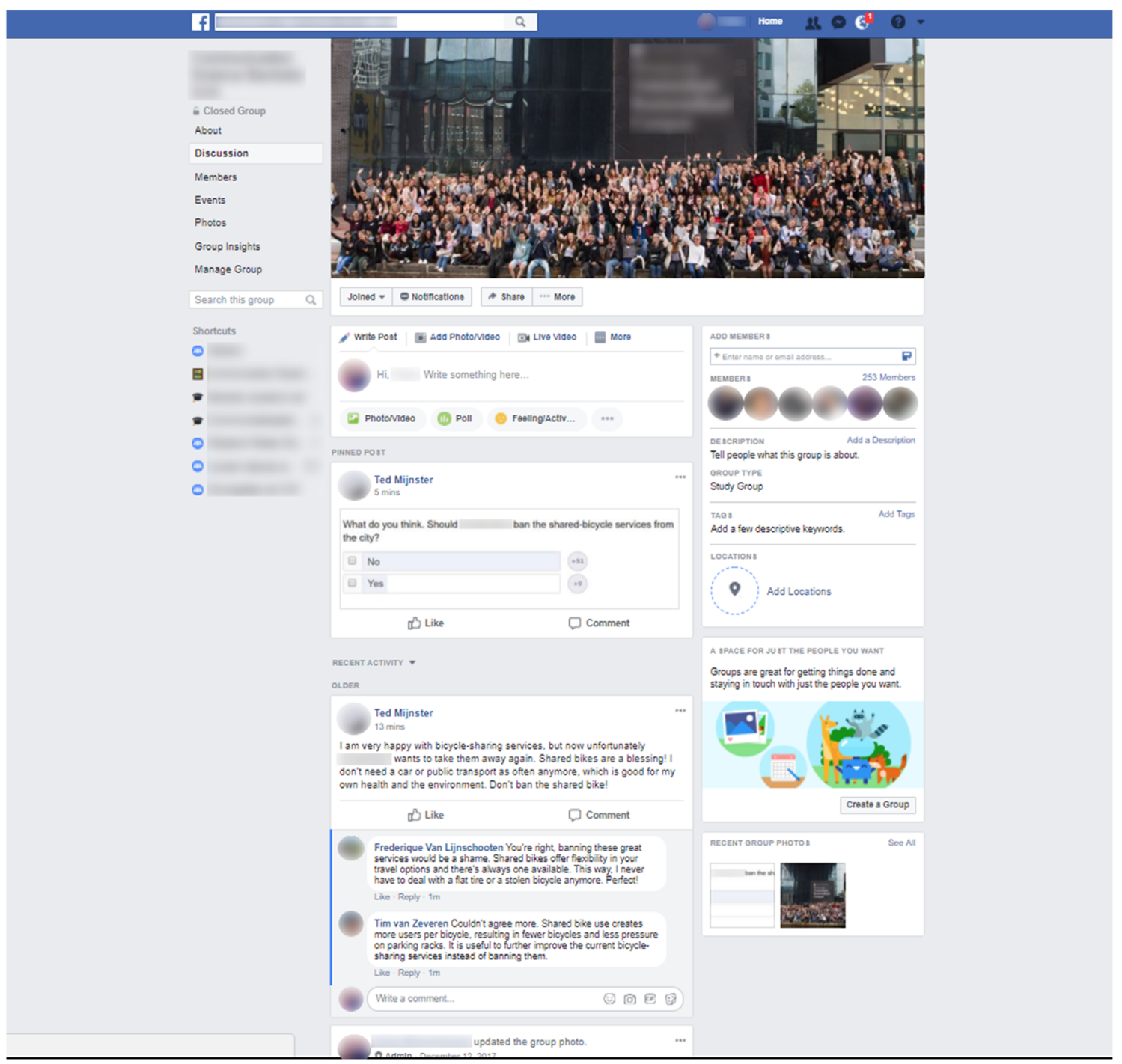

Figure 1. Screenshot of the Facebook group including poll and posts on the bicycle-sharing issue.

shown: "I read Amsterdam plans to ban bicycle-sharing services from the city. What are your opinions on that? Are you in favor or against the ban?" In the negative majority opinion conditions, the polls indicated that approximately $85 \%$ of the students were against the ban. Additionally, the post initiator indicated the following: "I am very happy with bicycle-sharing services, but now unfortunately Amsterdam wants to take them away again. Shared bikes are a blessing! I don't need a car or public transport as often anymore, which is good for my own health and the environment. Don't ban the shared bike!" - followed by two more commenters who agreed with the post initiator. In the positive majority opinion conditions, the poll showed that $85 \%$ of the students were in favor of the ban. In addition, the post initiator indicated the following: "Finally it seems Amsterdam is going to do something about those shared bikes that occupy too much public space. Huge amounts of them are rusting away in the city center without ever being used.
Time to get rid of them!" - followed by two more agreeing comments.

In the SNS conditions, the posts and comments were given by ostensible Facebook users whose profiles were created for the purpose of this study. All profiles had a significant number of friends, pictures, and personal details to ensure that the profiles were perceived as genuine. Comments in the online discussion forum were attached to nicknames (such as "Student22"). In the Word condition, no public profiles or nicknames were used.

\section{Measures}

\section{Overall Valence of the Expressed Opinion}

The overall valence of participants' opinion with regard to the ban was assessed as either negative $(-1 ; n=215)$, neutral $(0 ; n=11)$, or positive $(+1 ; n=76)$. All statements were coded by two research assistants. The intercoder reliability 
test with approximately $10 \%$ of the sample showed an agreement of Krippendorff's $\alpha=.87$. Participants' statements included an average of 79.81 words $(S D=47.50)$.

\section{Valence of Thoughts}

As a more fine-grained measure, each opinion was divided into thoughts (number of thoughts per statement: $M=4.79$, $S D=1.91$, which were detected by identifying separable statements, arguments, and suggestions. The thoughts were subsequently coded by two research assistants as positive, neutral, or negative $(\alpha=.90)$. The valence index of the expressed opinion reflected the relative valence of thoughts per participant. For this purpose, the difference between positive and negative thoughts was divided by the total number of thoughts $(M=-0.07, S D=0.19)$.

\section{Attitude Toward the Topic}

The dependent variable of participants' (private) attitude toward the topic was measured two times (before and after the opinion expression) on a scale from 1 (= strongly disagree) to 7 (= strongly agree) with three items: "Bicycle-sharing services in Amsterdam should be banned"; "Bicyclesharing services in Amsterdam are unacceptable"; and "The proposition to ban bicycle-sharing services in Amsterdam should be supported" (Cronbach's $\alpha$ at $\mathrm{T}_{1}=.86, M=$ $3.08, S D=1.34 ; \alpha$ at $\left.\mathrm{T}_{2}=.90, M=3.05, S D=1.44\right)$. Participants showed relatively high attitude certainty at $\mathrm{T}_{2}(M=$ $5.48, S D=1.23$; "How certain are you about your own opinion regarding the bicycle-sharing issue?" on a 7-point scale).

\section{Internalization}

To create the internalization scale, the attitude score at $\mathrm{T}_{1}$ was subtracted from the attitude score at $\mathrm{T}_{2}$. Participants who internalized an expressed positive opinion toward the ban should have an attitude at $T_{2}$ that is higher than at $\mathrm{T}_{1}$, while participants who internalized an expressed negative opinion should have an attitude at $\mathrm{T}_{2}$ that is lower than at $T_{1}$. To ensure that a higher score reflects a higher internalization score in both situations, the attitude $T_{2}$ minus $T_{1}$ score for participants who wrote down an overall negative statement about the ban was multiplied by -1 . The resulting internalization index could theoretically range from -6 to 6 and varied from -1.33 to 2.67 in the sample $(M=0.20$, $S D=0.66$ ). It should be noted that participants who were overall neutral in their statement with regard to the ban $(n=11)$ were filtered out when calculating the internalization index.

\section{Manipulation Checks}

\section{Perceived Valence of the Majority Opinion}

Participants evaluated the valence of the majority opinion as presented in the poll, posts, and comments on a 7-point scale from 1 (= extremely negative) to 7 (= extremely positive; $M=3.74, S D=2.12$ ).

\section{Public Identifiability}

To assess whether participants believed that their comment was visible to others, public identifiability was measured on a scale from 1 (= very publicly unidentifiable) to 7 (= very publicly identifiable) with the following item: "I think my comment with my personal opinion is ..." (Gonzales \& Hancock, 2008; $M=4.98, S D=1.36$ ).

\section{Members of the Facebook Groups}

Finally, to check whether participants recognized the members of the Facebook group they answered the following question: "Who were the members of the Facebook group that you just visited?" with either "Communication Science Bachelor students of the UvA," "Bachelor students of the UvA," "People from Amsterdam," or "I don't know." In addition, participants were asked about the likelihood that they will meet one of the members in the group in the future on a 7-point scale $(M=4.62, S D=1.74)$.

\section{Results}

\section{Randomization and Manipulation Checks}

The 12 experimental groups did not significantly differ with respect to participants' gender, age, education, interest in the bicycle-sharing issue, and prior usage of bikes and sharing services. Results of an ANOVA with the valence of the majority opinion and media context as independent variables and the perceived valence of the majority opinion as dependent variable showed a significant effect of the majority opinion manipulation, $F(2,237)=22.89, p<$ $.001, \eta_{\mathrm{p}}^{2}=.16,90 \% \mathrm{CI}[0.09,0.23]$, in the intended direction (positive: $M=4.81, S D=2.37$; no opinion: $M=3.67$, $S D=1.26$, negative: $M=2.79, S D=1.88$; Bonferroni post hoc comparisons all $p<.05)$. There was no significant effect of the media context manipulation and no significant interaction, indicating that the valence induction worked equally well across channels.

An ANOVA with media context and the valence of the majority opinion as independent variables showed that the perceived public identifiability of one's comment differed across the media conditions, $F(3,290)=3.69, p=$ $.012, \eta_{\mathrm{p}}^{2}=.04,90 \%$ CI [0.0045, 0.07]. Participants regarded their comments as more publicly identifiable in the general SNS student group with lower likelihood of future interaction $(M=5.27, S D=1.03)$ than in the forum $(M=4.63, S D=1.43$; Bonferroni comparison: $p=.025)$. The mean values for the SNS group with high likelihood of future interaction $(M=5.18, S D=1.40)$ and the Word 
Table 1. Contrast effects (Hypothesis 2): weights and descriptive statistics for effects of media context and majority opinion on the valence of participants' expressed opinion (thought valence index)

\begin{tabular}{|c|c|c|c|c|c|c|c|c|c|c|c|c|}
\hline \multirow{2}{*}{$\begin{array}{l}\text { Media context } \\
\text { Majority }\end{array}$} & \multicolumn{3}{|c|}{$\begin{array}{l}\text { SNS with high likelihood } \\
\text { of future interaction }\end{array}$} & \multicolumn{3}{|c|}{$\begin{array}{l}\text { SNS with low likelihood of } \\
\quad \text { future interaction }\end{array}$} & \multicolumn{3}{|c|}{ Online forum } & \multicolumn{3}{|c|}{$\begin{array}{c}\text { Anonymous text } \\
\text { document }\end{array}$} \\
\hline & Pos & Neg & No & Pos & Neg & No & Pos & Neg & No & Pos & Neg & No \\
\hline Majority & 1 & -1 & 0 & 1 & -1 & 0 & 1 & -1 & 0 & 1 & -1 & 0 \\
\hline Majority $\times$ media context & 1 & -1 & 0 & 1 & -1 & 0 & 0 & 0 & 0 & 0 & 0 & 0 \\
\hline Contrast total for $\mathrm{H} 2$ & 2 & -2 & 0 & 2 & -2 & 0 & 1 & -1 & 0 & 1 & -1 & 0 \\
\hline Valence of opinion (M) & 0.00 & -0.15 & -0.08 & -0.02 & -0.10 & -0.06 & -0.03 & -0.14 & -0.09 & 0.00 & -0.06 & -0.06 \\
\hline Valence of opinion (SD) & 0.22 & 0.18 & 0.19 & 0.18 & 0.13 & 0.14 & 0.22 & 0.34 & 0.19 & 0.15 & 0.13 & 0.16 \\
\hline
\end{tabular}

Note. SNS = social network sites; $\mathrm{H} 2$ = Hypothesis 2 .

document $(M=4.84, S D=1.47)$ were not significantly different from the other groups. All groups scored significantly higher than the scale midpoint, which was not expected for the private text document: One reason for this result could be that some participants expected researchers to read their statements and also interpreted this as public visibility (Walther et al., 2011).

A $t$ test with the media context as independent variable and the perceived likelihood of future interaction as dependent variable revealed no significant difference, $t(149)=$ $.717, p=.238$ (one-tailed; SNS group with high likelihood of interaction: $M=4.72, S D=1.73$; SNS group with low likelihood of interaction: $M=4.52, S D=1.77)$. This unexpected finding will be revisited in the "Discussion" section. However, results from a chi-square test with a cross-table of the experimental conditions (SNS with high vs. low level of future interaction) and the question about the members showed that most participants $(74 \%)$ were able to correctly recall the members of the Facebook group, $\chi^{2}(3)=52.30$, $p<.001$.

\section{Adaptation Effects}

In the first hypothesis, we proposed that the visible majority opinion affects the valence of participants' expressed opinion. An ANOVA was conducted with the valence of the majority opinion as independent variable and the thought valence index of the expressed opinion as dependent variable. The results showed that the direction of participants' opinion expression was significantly affected by the displayed majority opinion, $F(2,299)=7.21, p=.001, \eta^{2}=$ $.05,90 \%$ CI [0.01, 0.09]. Post hoc analyses with Bonferroni correction revealed that those exposed to a positive majority opinion $(M=-0.01, S D=0.19)$ were more positive toward the ban in their statement than those exposed to a negative majority opinion $(M=-0.11, S D=$ $0.21 ; p=.001)$, which partially supports $\mathrm{H} 1$. The positive condition did not significantly differ from the condition without a majority opinion $(M=-0.07, S D=0.17 ; p=$ .067). The difference between the negative majority condition and the no majority condition was not significant, either $(p=.409){ }^{1}$

In the second hypothesis, we posited that there would be a stronger adaptation of opinion expression toward the observed majority opinion in SNS compared to online forums or nonpublic channels. To test this, a planned contrast analysis with the assumed directional effects was performed. Due to the $4 \times 3$ between-subjects design, 12 contrast weights had to be specified, which were derived based on the effect of each factor in each condition. Subsequently, preliminary weights of all factors were summed in each cell (Rosenthal \& Rosnow, 1985; Walther et al., 2011). In the first step, to reflect the persuasive effect of the majority opinion, we assigned a preliminary weight of -1 for conditions with a negative majority opinion and a weight of +1 for conditions with a positive majority opinion. Since conditions with no majority opinion are not involved in the comparison, a weight of 0 was assigned to these groups. In the second step, the effect of the media context was taken into account. Since we expected the effect of the majority opinion to be stronger in the SNS conditions than in the forum and Word conditions, additional weights of -1 (in the cells with a negative majority opinion) and +1 (positive majority opinion) were assigned in the SNS conditions. Ultimately, the weights for each factor in each condition were summed to perform a contrast analysis to test the second hypothesis.

The contrast analysis with the thought valence index as dependent variable showed a significant effect, $t(133.71)=$ 3.04; $p=.003, r_{\text {contrast }}=.25$. However, the mean values do not fully adhere to the predicted patterns of the contrast weights (see Table 1): Even though the results suggested that there was a stronger adaptation of opinion expression toward the observed majority opinion in the SNS conditions with high likelihood of future interaction than in the forum

\footnotetext{
The valence index scores of the neutral and the negative majority opinion conditions were significantly different from $0, t(101)=-4.40, p<.001$ and $t(99)=-5.40, p<.001$.
} 
and text document conditions, differences between the SNS conditions with low likelihood of future interaction and the other conditions are not in line with the order of the weights. ${ }^{2}$ For this reason, $\mathrm{H} 2$ is not supported by the data.

\section{Internalization Effects}

To test the third hypothesis, in which we proposed that the expression of an opinion in an SNS context, compared to an online forum and a nonpublic setting, leads to stronger internalization of this opinion, a planned contrast analysis was conducted that compared the effect of the SNS conditions $(+1)$ with the other conditions $(-1)$. However, the analysis did not yield significant results, $t(279)=.82 ; p=.207$ (one-tailed), $r_{\text {contrast }}=.05^{3}$, meaning that $\mathrm{H} 3$ has to be rejected. An alternative way of testing internalization effects is to treat attitude at $T_{2}$ as the dependent variable and attitude at $\mathrm{T}_{1}$ as well as the valence of the expressed opinion as predictors in regression analyses. Attitude at $\mathrm{T}_{1}$ ( $\beta=.723 ; p<.001)$ and the thought valence index of the expressed opinion $(\beta=.242 ; p<.001)$ were found to be significant predictors of attitude at $\mathrm{T}_{2}$, while controlling for the media context in the regression analysis, $F(4,286)=$ $306.38 ; p<.001 ; R^{2}=.811,90 \%$ CI $[0.78,0.83]$, which supports the general notion that the content of opinion expression affected one's later attitude.

The fourth hypothesis posited that a high likelihood of expecting a future interaction with others within an SNS discussion group (students of the same major subject), as opposed to a low likelihood of future interaction in such a group, strengthens the internalization of the expressed opinion. To test this hypothesis, a planned contrast analysis was conducted that focused on the subsample of the SNS conditions. Hence, SNS conditions with high likelihood of future interaction $(+1)$ were compared with SNS conditions with low likelihood of future interaction $(-1)$, regarding the level of internalization. Results showed a significant contrast, $t(139)=1.94 ; p=.027$ (one-tailed), $r_{\text {contrast }}=.16$, and mean values indicate that SNS groups with a high likelihood of future interaction (positive valence: $M=0.25, S D$ $=0.55$, negative: $M=0.36, S D=0.69$, no majority opinion: $M=0.21, S D=0.70)$ resulted in stronger internalization than SNS groups with a low likelihood of future interaction (positive: $M=0.17, S D=0.80$, negative: $M=-0.15, S D=$ 0.50 , no majority opinion: $M=0.17, S D=0.65$ ). Therefore, $\mathrm{H} 4$ is supported by the data.

\section{Discussion}

The goal of this research was to investigate whether the characteristics of SNS increase the adaptation of expressed opinions to the majority and the internalization of these attitudes in comparison with other media contexts. Based on the literature on public self-presentation and identity shifts (Gonzales \& Hancock, 2008; Tice, 1992), it was assumed that the publicness of and identifiability within SNS elicit stronger expression effects than settings that are visually anonymous (online forums) or private (text-processing documents). A laboratory experiment was designed as a preliminary test of these predictions.

Concerning adaptation effects, participants' expressed opinions were significantly affected by the valence of the majority opinion, as visible in ostensible peer statements such as Facebook comments and a poll in the SNS conditions. This is in line with assumptions of general social influence of peers (Cialdini \& Goldstein, 2004) and studies that showed persuasive effects of online or SNS comments (Lee \& Jang, 2010; Winter et al., 2015); however, it has to be noted that the effect was small and only the comparison between positive and negative comments was significant. While the latter studies focused on readers' private attitudes, the present investigation showed effects of comments on the content of users' public statements. This can be explained with both informational and normative social influence (Deutsch \& Gerard, 1955) that cannot be fully disentangled without a condition in which there is no opinion expression at all.

Given the high salience of self-presentation in SNS (Nadkarni \& Hofmann, 2012) and a corresponding motivation to get along with others (Chaiken et al., 1996), we assumed that normative pressures and thus the public adaptation toward the majority opinion are stronger in SNS than in settings in which users are less identifiable. Although the mean values showed the highest orientation toward the majority in the SNS groups with students from the same subject, the overall prediction was not supported. Specifically, the adaptation in the SNS group with low likelihood of future interaction (students from the whole university) was not stronger than when participants wrote down their opinion in an online forum or a private text document. Particularly the latter comparison may suggest that the informational influence of peer statements is already quite high so that it also occurs in less identifiable settings (early studies on social influence in computer-mediated

\footnotetext{
${ }^{2}$ Similarly, an ANOVA with majority opinion and media context (clustered as SNS groups vs. non-SNS context) as independent variables did not show a significant interaction.

${ }^{3}$ The pattern of results remains the same when participants with extreme attitudes at T1 (a score of 1 or $7 ; n=20$ ) were filtered out in the internalization analyses (as their attitudes could not become more extreme).
} 
communication suggest that group memberships such as being a student may even be particularly influential in visually anonymous settings; Tanis \& Postmes, 2003) and may be amplified only in highly relevant SNS groups.

With regard to expression effects (Pingree, 2007; Valkenburg, 2017), we investigated whether people internalize the content of what they wrote down (for instance, when people who write something negative about the proposed bicycle-sharing ban become more negative in their [private] evaluation of the issue). The mean value of the internalization index was positive, and the valence of statements in the expressed opinion significantly predicted private attitudes after the act of expression, which corroborates the general notion of an expression effect (however, it has to be noted that there was no condition without any expression and the manipulation focused on the context in which the expression took place). Prior research on the internalization of displayed personality traits both offline (Schlenker et al., 1994; Tice, 1992) and online (Gonzales \& Hancock, 2008; Walther et al., 2011) showed larger identity shifts in public versus private settings. However, the present results did not show a generally higher internalization in SNS (in comparisons with setting with lower levels of public identifiability), which is not in line with the predictions derived from public commitment theory (Schlenker et al., 1994). This contrast might be explained by the difference between personality traits (that were the content of self-presentation in previous identity shift studies, mostly extraversion, which may also be partly confounded with the characteristics of publicness) and opinions on current issues (which were the focus of the present study).

Within the SNS conditions, a small significant effect occurred in that internalization was stronger in the SNS group of the same subject. This is in line with the more specific assumptions on publicness effects that highlight the likelihood of future interactions (Tice, 1992). However, it has to be mentioned that the manipulation check for this differentiation of high versus low likelihood of interaction did not reach significance. Perhaps the likelihood of meeting someone (in the question potentially interpreted as only in face-to-face settings) was not decisive for participants and it is conceivable that the common group membership (same-subject students vs. students from all subjects of the same university) was a more crucial factor. This needs to be disentangled in future studies. The second manipulation check, however, showed that most participants correctly remembered the name of the Facebook group, which indicates that the group difference was salient to them.

Concerning the question of whether the characteristics of social media strengthen expression effects, a tentative conclusion could be that relevant SNS audiences do so, but that internalization of opinions that were posted or written down also occurs in more conventional "Web 1.0" settings such as forums. Connecting the findings with the formation of public opinion and the flow of online discussions, it can be stated that there is a measurable influence of the majority opinion (or the predominant opinion in a given network) on what people believe and express in media channels and that the act of expression may solidify them or make them even more extreme. Increases in opinion expression might then strengthen popular opinions in the general public debate or in fragmented groups, which both could be problematic from a normative perspective (Steenbergen et al., 2003). As the present findings only show limited effects of the SNS audience, fears about such spiral processes in social media (that also require a clear opinion climate in the beginning) have to be hedged but cannot be completely dismissed. Perhaps the crucial difference between SNS and other less public settings is not the magnitude of expression effects but the frequency in which acts of expression take place: As SNS and other social media foster public opinion expression, expression effects become more likely (see Valkenburg, 2017), even though they could also occur in other settings. More work is needed to combine survey data on the frequency of opinion expression (e.g., with mobile experience sampling methods) with corresponding shifts. As the present investigation focused on Facebook discussion groups with peers, it is possible that even more relevant audiences (e.g., personally known Facebook friends) lead to stronger internalization. This could be addressed in future studies that, however, would face challenges with regard to experimental control and ethical issues.

One main contribution of the study is to extend research on online expression effects to the domain of opinions on current news and discussions: While prior research mainly employed the setting of self-presentation tasks with pre-defined goals (e.g., Carr \& Foreman, 2016; Gonzales \& Hancock, 2008), we argue that posting content on general news topics is a frequent activity with societal consequences that deserves further attention. Furthermore, the study offers a setting in which participants have many degrees of freedom on what to write. This is not only more realistic than acting in a prescribed way (e.g., highlighting one's extraverted sides), it also fulfils the condition of high choice in observable behavior, which Tice (1992) has named as an important factor of internalization effects, to a greater extent. Concerning the analytic strategy, the present method allows for a comparison of attitudes before and after opinion expression (pretest-posttest comparisons to detect actual shifts on within-person level), while previous work only considered between-condition differences in posttest variables. Although this likely leads to smaller effects, makes the analysis of internalization more complex (as it involves the consideration of the actual content of 
expression), and makes it more difficult to disentangle adaptation and expression effects, we believe that the higher external validity outweighs these disadvantages.

Nevertheless, the laboratory situation may still have appeared artificial to some participants: It is conceivable that those who felt a stronger need to adapt their opinions would refrain from opinion expression outside the lab; however, participants did not report any discomfort with the task during the experiment. Furthermore, the study is limited by a small sample size that was due to the availability of lab participants, which has resulted in low statistical power to detect small effects. Only one exemplary topic was selected, and to increase generalizability, findings should be replicated with further issues. Particularly for higher-involvement topics such as migration or the coronavirus pandemic, patterns may be different - for instance, with weaker adaptation effects when opinion certainty is high (Noelle-Neumann, 1974) but potentially stronger expression effects. We only employed specific prototypes of SNS (with a sub-variation of group composition) and online forums - for a more precise analysis of the underlying characteristics, a simultaneous variation of factors such as (visual) identifiability, connection with friends versus strangers, likelihood of future interaction, or relevance of group would be promising for further studies. Moreover, the experimental setting only reflects a short part in the process of online discussions: Feedback by other commenters (Walther et al., 2011) or the interplay between expression and reception effects (Valkenburg, 2017) could be studied in more process-oriented observations.

\section{Conclusion}

In summary, the present research suggests that people slightly adapt their opinion expression on current issues to the majority in CMC settings and tend to internalize these attitudes later. This internalization was stronger in SNS groups with a more relevant audience, which is in line with Tice's concept (1992) of future interaction, while the mere level of publicness and identifiability (Schlenker et al., 1994; Spears \& Lea, 1994) was not influential. Adaptation and expression effects were also visible in visually anonymous forums or after filling out a nonpublic document. But as the ease of expression in SNS and other genres of social media (Bayer et al., 2020) may still give prominence to these new platforms of public debates, further research is needed to specify the circumstances under which the characteristics of media settings may strengthen internalization. We hope that expressing these ideas in the present paper not only has an expression effect on ourselves but also exerts a classic reception effect in raising interest for the under-researched consequences of opinion expression.

\section{References}

Anonymous. (1998). To reveal or not to reveal: A theoretical model of anonymous communication. Communication Theory, 8, 381407. https://doi.org/10.1111/j.1468-2885.1998.tb00226.x

Baumeister, R. F., \& Tice, D. M. (1984). Role of self-presentation and choice in cognitive dissonance under forced compliance: Necessary or sufficient causes? Journal of Personality and Social Psychology, 46(1), 5-13. https://doi.org/10.1037/00223514.46.1.5

Bayer, J. B., Trieu, P., \& Ellison, N. B. (2020). Social media elements, ecologies, and effects. Annual Review of Psychology, 71(1), 471-497. https://doi.org/10.1146/annurev-psych-010419050944

Bem, D. J. (1972). Self-perception theory. Advances in Experimental Social Psychology, 6, 1-62. https://doi.org/10.1016/s00652601(08)60024-6

Boutyline, A., \& Willer, R. (2017). The social structure of political echo chambers: Variation in ideological homophily in online networks. Political Psychology, 38(3), 551-569. https://doi.org/ 10.1111/pops.12337

Boyd, D. M., \& Ellison, N. B. (2007). Social network sites: Definition, history, and scholarship. Journal of Computer-Mediated Communication, 13(1), 210-230. https://doi.org/10.1111/ j.1083-6101.2007.00393.x

Carr, C. T., \& Foreman, A. C. (2016). Identity shift III: Effects of publicness of feedback and relational closeness in computermediated communication. Media Psychology, 19(2), 334-358. https://doi.org/10.1080/15213269.2015.1049276

Chaiken, S., Giner-Sorolla, R., \& Chen, S. (1996). Beyond accuracy: Defense and impression motives in heuristic and systematic information processing. In P. M. Gollwitzer \& J. A. Bargh (Eds.), The psychology of action: Linking cognition and motivation to behaviour (pp. 553-578). Guilford Press.

Chaiken, S., Liberman, A., \& Eagly, A. H. (1989). Heuristic and systematic information processing within and beyond the persuasion context. In J.S. Uleman \& J. A. Bargh (Eds.), Unintended thought (pp. 212-252). Guilford Press.

Cialdini, R. B., \& Goldstein, N. J. (2004). Social influence: Compliance and conformity. Annual Review of Psychology, 55, 591622. https://doi.org/10.1146/annurev.psych.55.090902.142015

Deutsch, M., \& Gerard, H. B. (1955). A study of normative and informational social influence upon individual judgment. Journal of Abnormal and Social Psychology, 51(3), 629-636. https:// doi.org/10.1037/h0046408

Echterhoff, G., Higgins, E. T., Kopietz, R., \& Groll, S. (2008). How communication goals determine when audience tuning biases memory. Journal of Experimental Psychology, 137(1), 3-21. https://doi.org/10.1037/0096-3445.137.1.3

Festinger, L., \& Carlsmith, J. M. (1959). Cognitive consequences of forced compliance. Journal of Abnormal and Social Psychology, 58(2), 203-210. https://doi.org/10.1037/h0041593

Flesch, R. (1948). A new readability yardstick. Journal of Applied Psychology, 32(3), 221-233. https://doi.org/10.1037/h0057532

Gonzales, A. L., \& Hancock, J. T. (2008). Identity shift in computermediated environments. Media Psychology, 11(2), 167-185. https://doi.org/10.1080/15213260802023433

Higgins, E. T. (1992). Achieving "shared reality" in the communication game: A social action that creates meaning. Journal of Language and Social Psychology, 11(3), 107-131. https://doi. org/10.1177/0261927X92113001

Hogan, B. (2010). The presentation of self in the age of social media: Distinguishing performances and exhibitions online. Bulletin of Science, Technology \& Society, 30(6), 377-386. https://doi.org/10.1177/0270467610385893 
Johnson, B. K., \& van der Heide, B. (2015). Can sharing affect liking? Online taste performances, feedback, and subsequent media preferences. Computers in Human Behavior, 46, 181190. https://doi.org/10.1016/j.chb.2015.01.018

Lee, E.-J., \& Jang, Y. J. (2010). What do others' reactions to news on Internet portal sites tell us? Effects of presentation format and readers' need for cognition on reality perception. Communication Research, 37(6), 825-846. https://doi.org/10.1177/ 0093650210376189

McFarland, C., Ross, M., \& Conway, M. (1984). Self-persuasion and self-presentation as mediators of anticipatory attitude change. Journal of Personality and Social Psychology, 46(3), 529-540. https://doi.org/10.1037/0022-3514.46.3.529

Nadkarni, A., \& Hofmann, S. G. (2012). Why do people use Facebook? Personality and Individual Differences, 52(3), 243249. https://doi.org/10.1016/j.paid.2011.11.007

Neubaum, G., \& Krämer, N. C. (2018). What do we fear? Expected sanctions for expressing minority opinions in offline and online communication. Communication Research, 45(2), 139-164. https://doi.org/10.1177/0093650215623837

Noelle-Neumann, E. (1974). The spiral of silence. A theory of public opinion. Journal of Communication, 24(2), 43-51. https://doi. org/10.1111/j.1460-2466.1974.tb00367.x

Pingree, R. J. (2007). How messages affect their senders: A more general model of message effects and implications for deliberation. Communication Theory, 17(4), 439-461. https://doi. org/10.1111/j.1468-2885.2007.00306.x

Rosenthal, R., \& Rosnow, R. L. (1985). Contrast analysis: Focused comparisons in the analysis of variance. Cambridge University Press.

Schlenker, B. R., Dlugolecki, D. W., \& Doherty, K. (1994). The impact of self-presentations on self-appraisals and behavior: The power of public commitment. Personality and Social Psychology Bulletin, 20(1), 20-33. https://doi.org/10.1177/ 0146167294201002

Schlenker, B. R., \& Goldman, H. J. (1982). Attitude change as a self-presentation tactic following attitude-consistent behavior: Effects of choice and role. Social Psychology Quarterly, 45(2), 92-99. https://doi.org/10.2307/3033931

Spears, R., \& Lea, M. (1994). Panacea or panopticon? The hidden power in computer-mediated communication. Communication Research, 21(4), 427-459. https://doi.org/10.1177/ 009365094021004001

Steenbergen, M. R., Bächtiger, A., Spörndli, M., \& Steiner, J. (2003). Measuring political deliberation: A discourse quality index. Comparative European Politics, 1(1), 21-48. https://doi. org/10.1057/palgrave.cep.6110002

Tanis, M., \& Postmes, T. (2003). Social cues and impression formation in CMC. Journal of Communication, 53(4), 676-693. https://doi.org/10.1111/j.1460-2466.2003.tb02917.x

Thorson, K., \& Wells, C. (2016). Curated flows: A framework for mapping media exposure in the digital age. Communication Theory, 26(3), 309-328. https://doi.org/10.1111/comt.12087

Tice, D. M. (1992). Self-concept change and self-presentation: The looking glass self is also a magnifying glass. Journal of Personality and Social Psychology, 63(3), 435-451. https://doi. org/10.1037/0022-3514.63.3.435

Valkenburg, P. M. (2017). Understanding self-effects in social media. Human Communication Research, 43(4), 477-490. https://doi.org/10.1111/hcre.12113

Walther, J. B., Liang, Y., DeAndrea, D. C., Tong, S. T., Carr, C. T., Spottswood, E. L., \& Amichai-Hamburger, Y. (2011). The effect of feedback on identity shift in computer-mediated communication. Media Psychology, 14(1), 1-26. https://doi.org/10.1080/ 15213269.2010.547832
Winter, S., Brückner, C., \& Krämer, N. C. (2015). They came, they liked, they commented: Social influence on Facebook news channels. Cyberpsychology, Behavior, and Social Networking, 18(8), 431-436. https://doi.org/10.1089/cyber.2015.0005

\section{History}

Received December 10, 2019

Revision received May 5, 2021

Accepted May 7, 2021

Published online September 27, 2021

\section{Publication Ethics}

The study was approved by the local ethics committee.

\section{Open Data}

Data files and materials are available at the OSF repository: https://osf.io/5qf2r

\section{Funding}

The present study was funded by the German Research Foundation (Deutsche Forschungsgemeinschaft DFG; WI 4249/2-1). Open access publication enabled by the University of Koblenz-Landau.

\section{ORCID}

Stephan Winter

(D) https://orcid.org/0000-0002-8339-1937

\section{Stephan Winter}

Department of Psychology

University of Koblenz-Landau

Fortstr. 7

76829 Landau/Pfalz

Germany

stephan.winter@uni-landau.de

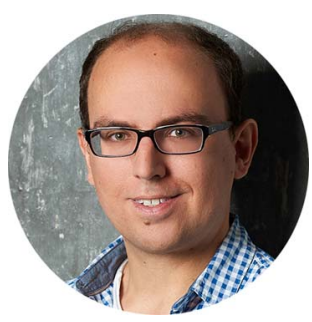

Stephan Winter (PhD, 2012) is Professor of Media Psychology at the University of Koblenz-Landau, Germany. His research interests include information selection as well as opinion formation and expression in online contexts. Previously, he was Assistant Professor of Persuasive Communication at the University of Amsterdam, The Netherlands.

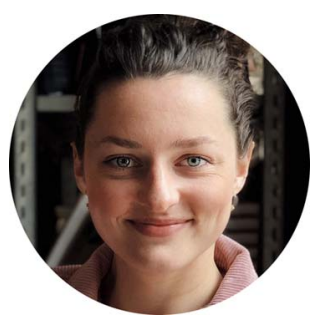

Anne Vos (MSc, 2019) is a PhD candidate at the Amsterdam School of Communication Research, University of Amsterdam, The Netherlands. Her research focuses on persuasive communication strategies as well as the integration of communication technologies and the promotion of health behavior change.

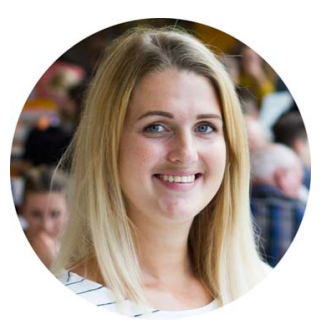

Paola Remmelswaal (MSc, 2017) is a former junior researcher of Persuasive Communication, University of Amsterdam, The Netherlands. She has worked on green advertising, creative media advertising, and new media uses and effects. 